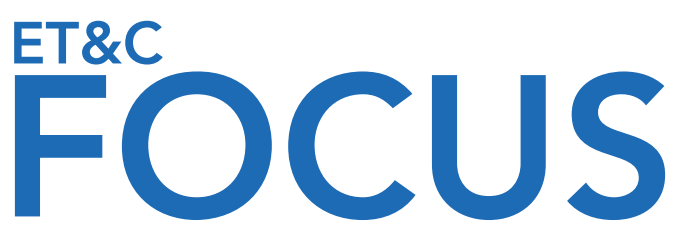

Focus articles are part of a regular series intended to sharpen understanding of current and emerging topics of interest to the scientific community.

\title{
Environmental Stressor Importance: Science versus Media
}

\author{
G. Allen Burton Jr.* and Eduardo Cimino Cervi \\ School for Environment and Sustainability, University of Michigan, Ann Arbor, Michigan, USA
}

It is well established that human perceptions of risk depend on a multitude of factors, such as subconscious processes, heuristics and biases, conscious reasoning, and social influences (Norgaard 2011). Even risk scientists are affected to some degree by nonscientific drivers, such as "known unknowns" and "unknown unknowns" (i.e., uncertainty). Another aspect affecting scientific investigations of environmental risk is media focus, which can influence research (Burton 2017). Given the daily news about climate change, natural disasters, endangered species, and chemical exposures, it is useful to understand public perceptions of risk-which are influenced by both science and media releases.

\section{METHODS}

\section{Environmental stressors}

To determine scientific, media, and public interest in climate change and other environmental stressors, we established relationships between research articles and media coverage from August 2018 to August 2019, and, through Google Trends, we also explored the volume of internet searches for selected environmental topics over the last $15 \mathrm{yr}$. Initially, 50 environmental topics were selected using Google search and Scopus (Figure 1). The criteria for selecting keywords were as follows: 1) words or phrases representing related queries for climate change on Google search and Scopus; 2) words or phrases with sufficient search volume in Google Trends to facilitate the analysis; 3) overlap with previous studies (Archibald and Butt 2018; Petersen et al. 2019) looking at online interest in environmental topics; and 4) words or short phrases that are specific and not prone to confusion with other popular, nonconservation search keywords (e.g., mercury the element may be used to refer to a famous singer or a planet in the solar system, so it was excluded).

\footnotetext{
This article contains online-only Supplemental Data.

* Address correspondence to burtonal@umich.edu

Published online 6 November 2019 in Wiley Online Library

(wileyonlinelibrary.com).
}

DOI: 10.1002/etc.4606
Although these keywords were not meant to form a comprehensive representation of conservation or environmental stressor topics, each keyword represents a relevant and common stressor or conservation issue illustrating our approach.

\section{Research articles database}

SJR SCImago Journal and Country Rank were used to select the 50 top-ranked scientific journals in the fields of "Ecology," "Environmental Engineering," "Environmental Sciences," and "Global and Planetary Change." An initial screening assessment was conducted to identify the pertinence of each journal for climate change research. Ten journals considered suboptimal for this evaluation were replaced with more suitable journals ranked in 51st to 100th SJR SCImago positions (see the Supplemental Data).

Using the Scopus database, a search was conducted to gather the total number of research articles related to all environmental topics. For each keyword, we used an advanced search tool to find articles published from August 2018 to August 2019 in all selected scientific journals, as follows: (ALL ("topic") AND SRCTITLE ("Journal X" OR "Journal Y" OR...) AND PUBYEAR $=$ 2018-2019).

\section{News website coverage}

News websites were selected using Alexa (Amazon), which hosts a global online electronic library of website traffic records. A total of 50 worldwide news websites were selected based on popularity (e.g., quantity of search traffic, high readership) and data accessibility (e.g., whether they can be accessed through databases such as LexisNexis, Access World News, Factiva, or their own archiving systems). The news websites selected were intended to cover the most representative countries across all continents. A search was conducted for news reported from August 2018 to August 2019 using each of the 50 environmental topics as the search term.

A search for online news was conducted using all environmental topics. For example, climate change was initially searched for in 8 English-speaking countries: Australia, Canada, England, India, Ireland, Scotland, South Africa, and the United 


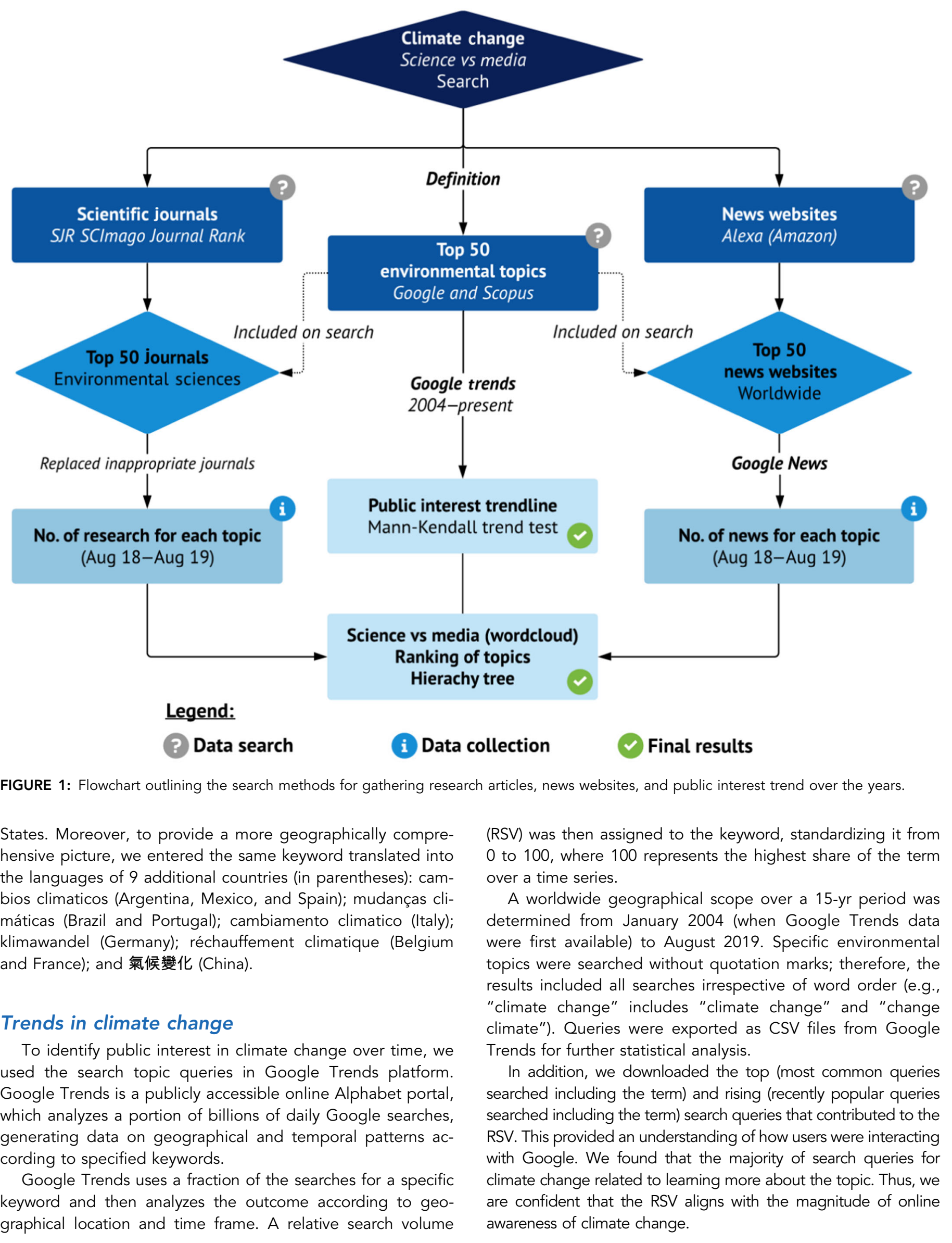




\section{Data analysis}

To provide a quick visual overview of search volumes for research articles and news websites related to environmental topics, we created 2-word clouds using the online website Wordclouds. The keyword global warming was considered an earlier synonym for climate change and was excluded in both word clouds.

The Mann-Kendall statistic $S$ test was applied to detect the presence of trends in public interest for each environmental topic over the year. Mann-Kendall tests the data against a null hypothesis of no trend and calculates Kendall's tau statistic $\tau$ based on $S$, the subtraction of the discordant pairs from the number of concordant pairs across all possible pairs in all the $n$ observations in the time series. A positive or negative value of $S$ (and thus $\tau$ ) indicates an upward or downward trend, respectively. Testing trends were investigated on a seasonal basis (12-mo period) at an $\alpha=0.05$ significance level using the $\mathrm{XLSTAT}^{\circledR}$ Premium package for Microsoft Excel ${ }^{\circledR}$.

\section{RESULTS AND DISCUSSION}

Climate change was the most popular topic reported in the public media last year (Figure 2), with over half a million news articles (average 12169/website). From pollution to corruption, environmental journalism often aims at highlighting problems and may fall into sensationalism to entice readership. This results in less focus on finding solutions, other causes of environmental impacts, or studies reporting an absence of adverse effects.
Climate change is fueling more frequent and intense precipitation, leading to more devastating flooding events. More than 3800 articles covering "flooding" appeared in the media last year, mostly describing flash flooding or devastation caused by hurricanes. The media focus on climate-related disasters (such as flooding, drought, and wildfires) tends to overlook nonclimate exacerbation of the impacts from public or government mismanagement of forests, water consumption, draining of wetlands, or environmental justice inequalities. By highlighting these factors, which contribute to the severity of climate-related disasters, the media could better educate both the public and environmental managers and policymakers.

The failure to report on all these related issues likely exacerbates public opinion divisions between liberal and conservative viewpoints. According to a CBS poll released in September 2019, whereas nearly 7 in 10 Democratic voters understand that humans significantly influence the climate and $80 \%$ want immediate action, just $20 \%$ of Republicans think humans are a primary cause, and barely $25 \%$ want rapid action (YouGov 2019).

Environmental news reporting also varies depending on the wealth of a particular country. Our results show that in developed countries, climate news tends to focus on scientific discoveries, controversies, and uncertainties. Among the media outlets, The New York Times and The Guardian had the highest coverage of environmental topics ( $2200 \mathrm{yr}^{-1}$ each) compared with the top 50 journals. Their stories primarily dealt with politics and regulations.

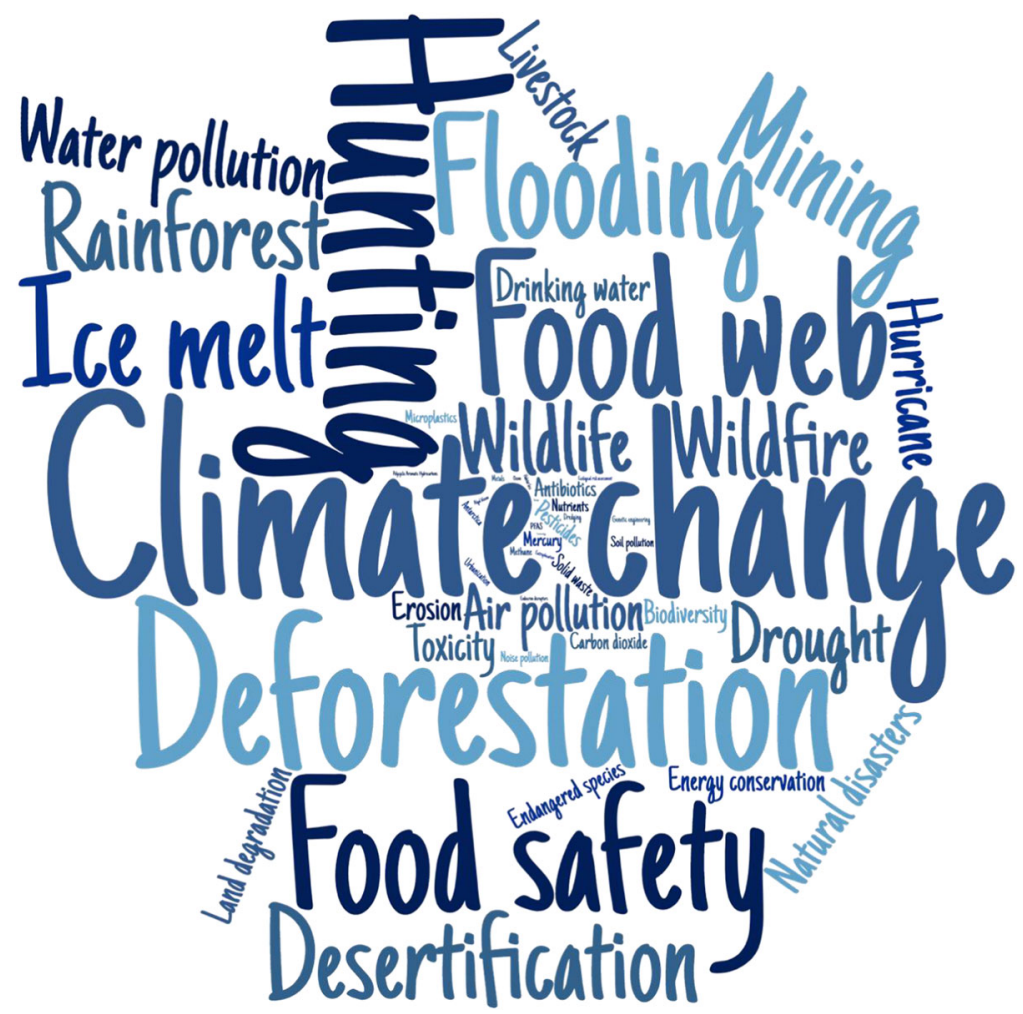

FIGURE 2: Top 50 environmental topics covered by news websites from August 2018 to August 2019. 
Climate change coverage in developing countries is similar in magnitude to that in developed countries; however, their reporting centers more on the economics of natural impacts_-such as deforestation and weather-related disasters. Deforestation and related fires in Brazil's Amazon rainforest were highlighted this year. From January to August 2019, 72000 wildfires in the Amazon area had been reported, an increase of $84 \%$ over the same period in 2018. More than 6000 total news reports globally included the term "deforestation" in 2019; $80 \%$ of the reports were during wildfire season (July and August). The Brazilian Amazon lost 430 sq. miles_-an area equivalent to Hong Kongin the first $26 \mathrm{~d}$ of August, according to preliminary data from the government's satellite monitoring agency; an area half the size of Philadelphia was reportedly lost in July (Brazilian National Institute for Space Research 2019).

"Climate change" was the second most popular topic reported by the scientific community (8971 total research articles), which is similar in importance to the public news media. According to a CBS poll, more than $25 \%$ of Americans consider climate change a "crisis," with a further $36 \%$ defining it as a "serious problem." Two in 10 respondents said it was a minor problem, with just $16 \%$ considering it not worrisome at all. Regardless of concern over climate change, there appears to be skepticism among Americans about how much humans can do about it. Just $19 \%$ said humans can stop rising temperatures and the associated impacts, with nearly $50 \%$ thinking it is possible to slow but not stop the changes and $23 \%$ refusing to believe humans can do anything at all (YouGov 2019).

Climate change scientists in developed countries focused their research keywords dealing with climate change on "regulation," "priority journals," and "animals," whereas scientists in developing countries focused their searches on the biological services and chemistry aspects of climate change. Thirty percent of the published research studies on climate change $(2642$ articles) were from developing countries (Argentina, Brazil, China, India, Mexico, and South Africa), and most of their keywords were "China," "nonhuman," "biodiversity," and "chemistry."

We were surprised that "hunting" was the second largest environmental subject reported on by the media. It was mainly related to African and American news, with more than 6000 reports last year. Nevertheless, a 2016 survey showed that only approximately $5 \%$ of Americans $16 \mathrm{yr}$ old and older actually hunt (US Department of the Interior, US Fish and Wildlife Service, and US Department of Commerce, US Census Bureau 2018). This low value is half of what it was $50 \mathrm{yr}$ ago, with accelerating declines expected over the next decade. The results also suggest that millennials are driving a global shift away from eating meat because of sensitivities over animal cruelty, and that young adults may be less likely to own guns than past generations (US Department of the Interior, US Fish and Wildlife Service, and US Department of Commerce, US Census Bureau 2018). The hunting news tended to focus on the decision to lift a hunting ban on elephants in some African countries.

The least popular topic in scientific reporting was "noise pollution," whereas in the public media it was "ecotoxicology." Only 1 news article/yr (average of 50 websites) emphasized the importance of linking the toxic effects of chemicals on ecosystems and their organisms. Even though our humandominated ecosystems receive many thousands of chemicals through atmospheric, terrestrial, and aquatic discharges, there is little focus on these exposures to wildlife and how climate change accentuates their impacts. This lack of public education raises the question of whether this linkage between chemical toxicity and climate change is simply too complicated to report. However, we believe the public would be interested in learning more about this aspect of climate change, such as how chemical exposures to both humans and wildlife dramatically increase following disasters.

Despite the high level of confidence the public has in science, scientists may not deliver convincing or effective messages to educate journalists and the public about climate-related risks-which are often complex and tied to many other issues such as poor zoning, inadequate infrastructure, and loss of natural buffers such as wetlands, sand dunes, and mangroves.

As an example, last year's most popular topic in scientific publishing was "metals," with more than 13500 research articles (Figure 3); however, metals are of little interest to the public or the media. So why this emphasis on metals? Perhaps it is because metals are common in our environment and everyday life, and are frequently associated with environmental contamination. It may also be related to the fact that metals are less expensive and easier to measure than high-profile organic chemicals, such as polychlorinated biphenyls, dioxin, per- and polyfluoroalkyl substances (PFAS), and endocrine disruptors. This allows limited research funding to stretch further, particularly for scientists in developing countries.

Our evaluation of public interest in environmental stressors (all searches) is shown in Figure 4. Google Trends shows how search topics trend up and down since 2004. For example, there has been a significant upward trend (0.82) in searches for "antibiotics" since 2004, probably because of a growing awareness (often through scientific publications) of the presence of antibiotics in our foods, waterways, and wastewaters, and the danger of increasingly drug-resistant pathogens.

Ozone depletion and global warming were at the top of environmental concerns in the 1970s and 1980s. Searches for both of these subjects have since declined. The search for "global warming" began to decline $(\tau=-0.68)$ after 2004 , as public interest in the term "climate change" $(0.25)$ increased. One possible reason for the decline may be that the term "climate change" is more correct as a descriptor than "global warming," because it encompasses all changes in the global climate (such as increasing cold and heat extremes).

Earlier this year, The Guardian updated its style guide to introduce new terms that more accurately describe the environmental crises facing the world. It now favors the terms "climate crisis" and "climate emergency" over "climate change." Earlier this year, The Guardian also began putting global $\mathrm{CO}_{2}$ levels into the daily weather forecast in its print publication.

Access to fresh water is a major driver of social conflict in developing countries-and as both population and vulnerability to climate change have increased, so has public interest. Water policies have profound impacts on food, land, wildlife, 


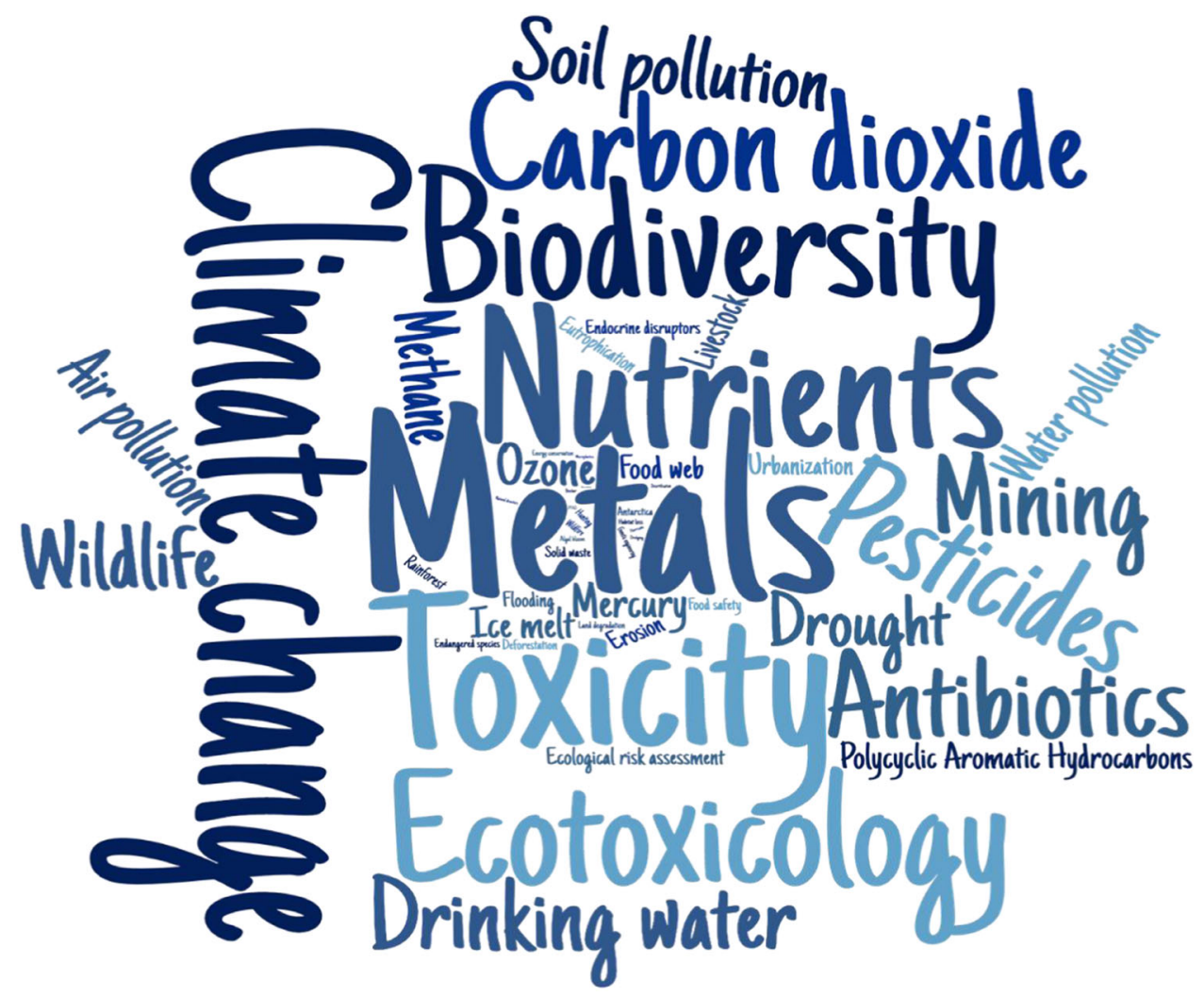

FIGURE 3: Top 50 environmental topics published in top 50 scientific journals from August 2018 to August 2019.

and relationships with neighboring countries. At the same time, multinational corporations are seeking new supplies in some of the world's thirstiest places. The RSV for "drinking water" doubled over the past decade, from 45 to 90 from 2009 to 2019, with an average $\tau=0.64$ (2004 to present).
Over the past decade, compounds in the class known as PFAS have emerged as major water contaminants around the globe. The RSV for PFAS was less than 10 until 2016, but has now dramatically increased (to 81 in 2019). The true risk to humans and wildlife of the myriad of PFAS chemicals is highly

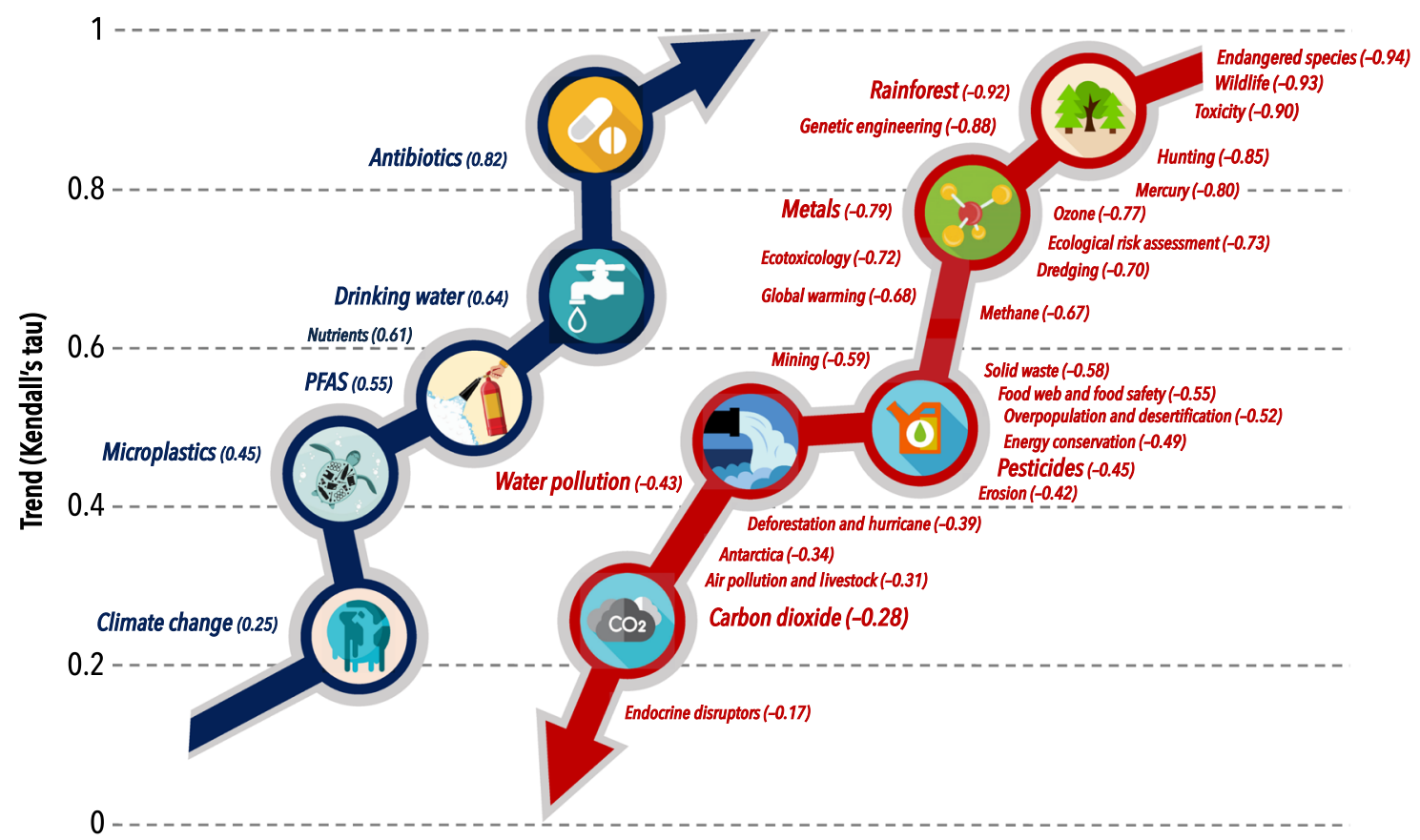

FIGURE 4: Public trends in Google searches of the top 50 environmental topics from 2004 to present. PFAS = per- and polyfluoroalkyl substances. 
uncertain, and only recently have significant efforts been directed toward increasing our understanding of the impacts of these compounds (US Environmental Protection Agency 2019). Overall, the searches covered by Google Trends seem to be more influenced by media clamor.

Another example of rapidly increasing attention is "microplastics," which we now know are pervasive around the globe. Microplastics showed a significant upward trend $(\tau=0.45)$ in search interest from 2004 to the present, with most searches being in the last $5 \mathrm{yr}$. The environmental threats to aquatic organisms and humans from microplastics have been a common news item. The media, advocacy groups, and some scientists have clamored over their prevalence and risks to humans and wildlife. Despite the paucity of field data showing impacts on populations and communities (e.g., Science Advice for Policy by European Academies 2019), a minor component of microplastics (microbeads) has been banned in many countries. There has been no call to ban the dominant form of microplastic, fibers, which primarily originate from washing our clothes. Some well-meaning scientists have perhaps overstated the risks of microplastics by not taking into account environmentally realistic exposure concentrations. The PFAS and microplastic examples are but 2 that point to a need for better science, more research, and better communication of the science to journalists, the public, and policymakers.

Recently, Our World in Data (2019) performed a comparison of actual causes of death in 2016, share of Google searches, and share of media coverage. They showed dramatic differences between reality and media coverage: more than $33 \%$ of all news was on terrorism (actual deaths less than 1\%), with approximately $15 \%$ on cancer and heart disease (actual deaths $~ 60 \%$ ). Also, what Americans search for on Google is a much closer reflection of what kills us than what is presented in the media. One way to think about it is that media outlets may produce content they think readers are most interested in, but such content is not necessarily reflected in our preferences when we search for information ourselves (Our World in Data 2019). It is well known that risk perceptions are often dominated by our most recent experiences, including what we hear. In a time of limited, even shrinking, research funding, it is critical that we focus the limited resources on increasing our understanding of the exposures to and possible adverse effects from the most important environmental stressors in our rapidly changing world.

It often seems that environmental scientists have little impact on the views of the public and policymakers. When issues are complicated, particularly if they are politically charged, it is difficult to meet the need of journalists and the media to attract attention and viewers. One success story, however, was the effective communication on the dire consequences of a declining ozone layer in the 1970s and 1980s. The media captured the attention of the public and policymakers using a simple story: There's a hole in the atmosphere's protective layer; without this layer, the risk of skin cancer greatly increases, and the chlorofluorocarbons in aerosols are primarily responsible. The public and policymakers responded, and the ozone is re-establishing.

At the end of April 2019, the Columbia Journalism Review and The Nation launched "Covering Climate Now," a project aimed at encouraging news organizations to raise their game when it comes to climate coverage. The Covering Climate Now goal was to gather all climate stories under one umbrella in the lead-up to the United Nations Climate Summit on 23 September 2019. The project included more than 250 outlets worldwide, and dozens of institutional and independent partners, with a combined audience of more than 1 billion people.

So, what is our message? First, large important environmental problems that hurt humanity and wildlife need a simple storyline, offered with both short- and long-term solutions so the public and policymakers can respond effectively. Scientists are the critical component: they define the problem, the solutions, and the communication with the media and policymakers. The media need a clear story of risks occurring locally, not just in far-away places to people we do not know. Also, our scientific preoccupation with complex models and uncertainty has consequences that are opposite to our hopes. People need solutions, and these must include simple, local options, not just countrywide emission goals, carbon taxes, and complaining about fossil fuels. Reducing our use of environmental stressors in everyday life may not seem like much compared with the role of China and India in climate change-but it forms the building blocks for greater change, future policy, and the lives of our children.

Data Accessibility_Please contact the corresponding author (burtonal@umich.edu) for any requests for access to data.

\section{REFERENCES}

Archibald CL, Butt N. 2018. Using Google search data to inform global climate change adaptation policy. Clim Change 150:447-456.

Brazilian National Institute for Space Research. 2019. Earth Observation General Coordination. Monitoring Program of The Amazon and Other Biomes. Deforestation-Legal Amazon. São José dos Campos, Brazil. [cited 2019 September 24] Available from: http://terrabrasilis.dpi.inpe. br/downloads

Burton GA Jr. 2017. Stressor exposures determine risk: So, why do fellow scientists continue to focus on superficial risks of popular topics? Environ Sci Technol 51:13515-13516.

Norgaard KM. 2011. Living in Denial: Climate Change, Emotions, and Everyday Life. MIT, Cambridge, MA, USA.

Our World in Data. 2019. Does the news reflect what we die from? Global Change Data Lab, University of Oxford, Oxford, UK. [cited 2019 September 14] Available from: https://ourworldindata.org/does-thenews-reflect-what-we-die-from

Petersen AM, Vincent EM, Westerling AL. 2019. Discrepancy in scientific authority and media visibility of climate change scientists and contrarians. Nat Commun 10:3502.

Science Advice for Policy by European Academies. 2019. A scientific perspective on microplastics in nature and society. Berlin, Germany. [cited 2019 September 14]. Available from: DOI: 10.26356/microplastics

US Department of the Interior, US Fish and Wildlife Service, and US Department of Commerce, US Census Bureau. 2018. 2016 National Survey of Fishing, Hunting, and Wildlife-Associated Recreation. Washington, DC.

US Environmental Protection Agency. 2019. EPA's per- and polyfluoroalkyl substances (PFAS) action plan, EPA 823R18004. Final/Technical Report. Washington, DC.

YouGov. 2019. Climate change will be an issue for most voters in 2020. CBS News, New York, NY, USA. [cited 2019 September 14] Available from: https://www.cbsnews.com/news/cbs-news-poll-climate-change-will-be-anissue-for-most-voters-in-2020 